\title{
Comorbidity, Frailty, and Waitlist Mortality among Kidney Transplant Candidates of All Ages
}

\author{
María Pérez Fernández ${ }^{a}$ Patricia Martínez Miguel ${ }^{\mathrm{a}}$ Hao Ying ${ }^{\mathrm{b}}$ \\ Christine E. Haugen $^{b}$ Nadia M. Chu ${ }^{c}$ Diego María Rodríguez Puyola \\ Leocadio Rodríguez-Mañas $^{d}$ Silas P. Norman ${ }^{\mathrm{e}}$ Jeremy D. Walston ${ }^{f}$ \\ Dorry L. Segev b, c Mara A. McAdams-DeMarcob, c
}

\begin{abstract}
a Department of Nephrology, Hospital Príncipe de Asturias, Alcalá de Henares, Madrid, Spain; ${ }^{b}$ Department of Surgery, Johns Hopkins University School of Medicine, Baltimore, MD, USA; ' Department of Epidemiology, Johns Hopkins School of Public Health, Baltimore, MD, USA; ${ }^{d}$ Hospital Universitario de Getafe, Madrid, Spain; ${ }^{e}$ Division of Nephrology, Department of Internal Medicine, University of Michigan School of Medicine, Ann Arbor, MI, USA; fDepartment of Medicine, Johns Hopkins University School of Medicine, Baltimore, MD, USA
\end{abstract}

\section{Keywords \\ Frailty $\cdot$ Comorbidity $\cdot$ Kidney transplantation}

\begin{abstract}
Background: Kidney transplantation (KT) candidates often present with multiple comorbidities. These patients also have a substantial burden of frailty, which is also associated with increased mortality. However, it is unknown if frailty is merely a surrogate for comorbidity, itself an independent domain of risk, or if frailty and comorbidity have differential effects. Better understanding the interplay between these 2 constructs will improve clinical decision making in KT candidates. Objective: To test whether comorbidity is equally associated with waitlist mortality among frail and nonfrail KT candidates and to test whether measuring both comorbidity burden and frailty improves mortality risk prediction. Methods: We studied 2,086 candidates on the KT waitlist (November 2009 - October 2017) in a multicenter cohort study, in whom frailty and comorbidity were measured at evaluation. We quantified the association between Charl-
\end{abstract}

son comorbidity index $(\mathrm{CCl})$ adapted for end-stage renal disease and waitlist mortality using an adjusted Cox proportional hazards model and tested whether this association differed between frail and nonfrail candidates. Results: At evaluation, $18.1 \%$ of KT candidates were frail and $51 \%$ had a high comorbidity burden ( $\mathrm{CCl}$ score $\geq 2)$. Candidates with a high comorbidity burden were at 1.38 -fold $(95 \% \mathrm{Cl} 1.01-$ 1.89 ) increased risk of waitlist mortality. However, this association differed by frailty status ( $p$ for interaction $=0.01$ ): among nonfrail candidates, a high comorbidity burden was associated with a 1.66 -fold $(95 \% \mathrm{Cl} 1.17-2.35)$ increased mortality risk; among frail candidates, here was no statistically significant association (HR 0.75, 95\% Cl 0.44-1.29). Adding this interaction between comorbidity and frailty to a mortality risk estimation model significantly improved prediction, increasing the c-statistic from 0.640 to 0.656 ( $p<$ $0.001)$. Conclusions: Nonfrail candidates with a high comorbidity burden at KT evaluation have an increased risk of waitlist mortality. Importantly, comorbidity is less of a concern in already high-risk patients who are frail.

(c) 2019 S. Karger AG, Base

\section{KARGER}

(C) 2019 S. Karger AG, Basel

E-Mail karger@karger.com

www.karger.com/ajn
Mara A. McAdams-DeMarco, $\mathrm{PhD}$

Department of Epidemiology

615 , N. Wolfe Street, W6033

Baltimore, MD 21205 (USA)

E-Mail mara@jhu.edu 


\section{Introduction}

Patients with end-stage renal disease (ESRD) often present with multiple comorbidities, which influence the progression of their ESRD, as well as survival $[1,2]$. Assessment of comorbidities is particularly important among ESRD patients who are being evaluated for kidney transplantation (KT) and often summarized through comorbidity indices such as the Charlson comorbidity index (CCI), which has been adapted for patients with ESRD [3,4]. A higher burden of pre-KT comorbidities is associated with increased risk of post-KT mortality [5] and surgical complications [6-8]. Therefore, it is likely that a high comorbidity burden is also associated with waitlist mortality among KT candidates; a better understanding of this association may help improve the selection of KT candidates [7, 8].

Frailty, a phenotype of decreased physiologic reserve resulting in vulnerability to stressors [9-11], was originally identified in community-dwelling older adults [9] and is associated with mortality and adverse outcomes [9, $10,12,13]$. Frailty is often incorrectly viewed as a surrogate for comorbidity [14]; however, they are 2 clearly differentiated phenotypes [15]. Frailty is a syndrome resulting from age-related physiologic changes (primary frailty) but can also arise secondary to chronic diseases (secondary frailty). In ESRD patients of all ages, frailty is common and an independent risk factor for mortality [16-18]. In this population, in which there is a high burden of comorbidity, it is unknown if frailty is merely a surrogate for comorbidity or whether they capture unique domains of risk. The differential effects of comorbidity on waitlist mortality between frail and nonfrail candidates are unclear but likely given the differential effects of frailty among hemodialysis patients by obesity status [19] and among KT recipients by depressive symptoms [20].

The main goals of this study were to (1) ascertain the burden of comorbidity using the CCI among frail and nonfrail KT candidates and (2) test whether comorbidity was associated with waitlist mortality in both frail and nonfrail KT candidates. In addition to quantifying the differential impact of the comorbidity burden on waitlist mortality between frail and nonfrail KT candidates, we estimated the improvement in waitlist mortality risk prediction provided by comorbidity and frailty.

\section{Materials and Methods}

\section{Study Design}

This was a prospective, multicenter, longitudinal cohort study of 2,086 adult patients (aged 18 and older) who were evaluated for
KT and listed at Johns Hopkins Hospital, Baltimore, MA, USA, between November 2009 and October $2017(n=1,986)$ and the University of Michigan, Ann Arbor, MI, USA, between January 2015 and April $2016(n=100)$. In this study, we measured frailty at the time of KT evaluation, as described below. Additional participant characteristics, including comorbidity (as described below), were also assessed at this time or abstracted from the KT evaluation medical record (cause of ESRD, blood type, time on dialysis, type of dialysis, and previous transplant). Demographics (age, gender, and race) were obtained by participant self-report, and body mass index (BMI) was calculated from self-reported height and dry weight. The Johns Hopkins Institutional Review Board and the University of Michigan Institutional Review Board approved the study, and all participants provided written informed consent. This research is in adherence with the Declaration of Helsinki and the Declaration of Istanbul.

\section{Comorbidity Burden}

Comorbidities from the ESRD-specific CCI [4], a validated index that takes into account both the number and seriousness of comorbid disease in patients with ESRD, were ascertained from medical records and/or self-reporting a history of cardiovascular disease (myocardial infarction, congestive heart failure), peripheral vascular disease, cerebral vascular disease, dementia, chronic lung disease, rheumatologic disorders, peptic ulcer, diabetes with (ESRD secondary to diabetes, patients with diabetic neuropathy, or diabetic retinopathy) or without complications, moderate or severe liver disease, metastatic cancer, leukemia, lymphoma, and HIV. We calculated the CCI as has been previously described [4]. We defined high comorbidity burden as a CCI score of 2 or more and low comorbidity burden as a score of 0 or 1 .

\section{Frailty Measurement}

At evaluation for KT, frailty was measured as defined and validated by Fried et al. [9] among a population of community-dwelling older adults, as well as by our group in ESRD and KT populations [16-18, 21-31]. Frailty is a phenotype based on 5 components: shrinking (self-report of unintentional weight loss of more than 10 pounds in the past year based on dry weight), weakness (grip strength below an established cutoff based on gender and BMI), exhaustion (self-report), low activity (kilocalories per week below an established cutoff), and slowness (walking time of 15 feet below an established cutoff by gender and height) [9]. Each of the 5 components was scored as 0 or 1 representing the absence or presence of that component. The aggregate frailty score was calculated as the sum of the component scores (range 0-5). Nonfrail patients were defined as a score of 0 , intermediate frailty patients were defined as a score of 1 or 2 , and frail patients were defined as a score of 3 or higher.

\section{Waitlist Mortality}

All participants were evaluated for KT and on the KT waitlist at Johns Hopkins University and University of Michigan. Participants were followed from the date of listing (index date) to December 1, 2017, when they were administratively censored. We censored participants who received KT, as is standard in the SRTR models for waitlist mortality (33). Mortality ascertainment was augmented with linkage to the Social Security Death Master File. 
Statistical Analysis

We quantified the risk of waitlist mortality associated with a 1 -point increase in the CCI score using Cox proportional hazards models; participants were followed from the date of listing to transplantation, death, or administrative censoring (December 1, 2017). For all models, we adjusted for factors included in the registry-based model (age, race, sex, blood type, and cause of ESRD). Then we tested whether this measure of comorbidity had a differential association with waitlist mortality between frail and nonfrail candidates by including an interaction term between CCI and frailty; statistical significance was estimated using a Wald test and a cutoff of $\alpha<0.05$. We used a similar approach defining a dichotomous indicator of high comorbidity burden (score of 2 or higher on the CCI).

We then calculated discrimination ability of the registry-based waitlist mortality model using Harrell's c-statistic, a measure of concordance indicating the model ability to discriminate between candidates with different times until death [32]. The c-statistic ranges from 0.5 (no discrimination) to 1.0 (perfect discrimination), and higher values indicate that the model more accurately predicts which patients will have longer survival. We then added the CCI score and high comorbidity burden as well as the synergistic effects of these with frailty and older age (age $\geq 65$ years) separately to the registry-based waitlist mortality model and calculated the c-statistic for each model.

\section{Results}

\section{Study Population}

Among 2,086 KT candidates, the mean age was 53.8 years old (SD 13.5), 40.1\% were female, $44.0 \%$ were African American, 45.9\% were obese (BMI $>30 \mathrm{~kg} / \mathrm{m}^{2}$ ), and $18.1 \%$ were frail at the time of KT evaluation. Additionally, $46.9 \%$ of participants were on hemodialysis, and $40.8 \%$ had not initiated renal replacement therapy.

\section{Comorbidity Burden among KT Candidates}

At the time of KT evaluation, the median CCI score was 2 (interquartile range [IQR] $0-3$ ); 51\% of the population had 2 or more comorbidities. The most common comorbid conditions from the CCI were diabetes $(38.6 \%)$, diabetes with complications (25.2\%), and congestive heart failure (14.4\%).

KT candidates with a high comorbidity burden were more likely to be older (age $\geq 65$ years) than candidates without a high comorbidity burden at the time of KT evaluation ( 28.5 vs. $19.0 \%$; $p<0.001$; Table 1$)$. Moreover, patients with a high comorbidity burden were less likely to be female ( 36.0 vs. $44.4 \% ; p<0.001$ ), but were more likely to be African American (49.4 vs. 38.4\%; $p<0.001$ ), obese (55.6 vs. $35.8 \% ; p<0.001$ ), and have an activities of daily living (ADL) disability (6.2 vs. $2.7 \%$; $p<0.001)$ or instrumental activities of daily living disability (22.2 vs.
Table 1. Characteristics of the KT candidates $(n=2,086)$ by CCI score. The CCI is modified for ESRD patients, and we defined a high comorbidity burden as a CCI score of $\geq 2$ and low comorbidity burden as a CCI score of 0 or 1 . Percentages are presented in the table

\begin{tabular}{|c|c|c|c|}
\hline Characteristic & $\begin{array}{l}\text { Low } \\
\text { comorbidity } \\
\text { burden } \\
(n=1,023)\end{array}$ & $\begin{array}{l}\text { High } \\
\text { comorbidity } \\
\text { burden } \\
(n=1,063)\end{array}$ & $p$ value \\
\hline Age $\geq 65$ years old & 19.0 & 28.5 & $<0.001$ \\
\hline Female & 44.4 & 36.0 & $<0.001$ \\
\hline Black & 38.4 & 49.4 & $<0.001$ \\
\hline Hispanic & 2.5 & 1.8 & 0.24 \\
\hline \multicolumn{4}{|l|}{ BMI, $\mathrm{kg} / \mathrm{m}^{2}$} \\
\hline$<25$ & 32.8 & 18.2 & \multirow[t]{3}{*}{$<0.001$} \\
\hline $25-29$ & 31.5 & 26.3 & \\
\hline$\geq 30$ & 35.8 & 55.6 & \\
\hline \multicolumn{4}{|l|}{ Blood type } \\
\hline A & 31.5 & 33.0 & \multirow[t]{4}{*}{0.21} \\
\hline B & 5.0 & 3.2 & \\
\hline $\mathrm{AB}$ & 16.6 & 16.9 & \\
\hline $\mathrm{O}$ & 46.9 & 46.9 & \\
\hline \multicolumn{4}{|l|}{ Dialysis type } \\
\hline $\mathrm{HD}$ & 42.7 & 51.1 & \multirow[t]{3}{*}{0.002} \\
\hline $\mathrm{PD}$ & 12.3 & 12.3 & \\
\hline Not on dialysis & 45.1 & 36.6 & \\
\hline \multicolumn{4}{|c|}{ Time on dialysis, years } \\
\hline 0 & 45.0 & 36.3 & \multirow[t]{3}{*}{$<0.001$} \\
\hline $1-2$ & 31.0 & 42.3 & \\
\hline$>2$ & 24.1 & 21.4 & \\
\hline ADL disability & 2.7 & 6.2 & $<0.001$ \\
\hline IADL disability & 11.5 & 22.2 & $<0.001$ \\
\hline \multicolumn{4}{|l|}{ Cause of ESRD } \\
\hline Glomerular & 29.9 & 12.1 & \multirow[t]{5}{*}{$<0.001$} \\
\hline Diabetes & 1.4 & 41.1 & \\
\hline Hypertension & 31.9 & 28.6 & \\
\hline Cystic diseases & 12.4 & 2.7 & \\
\hline Other & 24.4 & 15.4 & \\
\hline
\end{tabular}

KT, kidney transplantation; CCI, Charlson comorbidity index; ESRD, end-stage renal disease; BMI, body mass index; ADL, activities of daily living; IADL, instrumental activities of daily living.

$11.5 \% ; p<0.001)$. In addition, KT candidates with a high comorbidity burden were more likely to be undergoing hemodialysis (51.1 vs. $42.7 \%$; $p=0.002$ ). However, those with comorbidity had less time on dialysis (1.5 vs. 1.3 years; $p=0.04)$.

\section{Frailty and Comorbidity Burden}

There was some but not complete overlap of frailty, ADL disability, and a high comorbidity burden at the time of KT evaluation (Fig. 1). Among the KT candidates, 
Fig. 1. Overlap of frailty, disability, and comorbidity among KT candidates of all ages. The CCI is modified for ESRD patients and we defined a high comorbidity burden as a CCI score of $\geq 2$ and low comorbidity burden as a CCI score of 0 or 1 . CCI, Charlson comorbidity index; ADL, activities of daily living.



Table 2. CCI and the components of the CCI by frailty status among KT candidates $(n=2,086)$

\begin{tabular}{lcccc}
\hline Comorbidities & $\begin{array}{c}\text { Nonfrail } \\
(n=396)\end{array}$ & $\begin{array}{c}\text { Intermediately } \\
\text { frail }(n=1,312)\end{array}$ & $\begin{array}{l}\text { Frail } \\
(n=378)\end{array}$ & $p$ value \\
\hline CCI score, median (IQR) & $0(0.2)$ & $2(0.3)$ & $2(0.4)$ & $<0.001$ \\
Myocardial infarction & 5.8 & 7.9 & 7.9 & 0.36 \\
Peripheral vascular disease & 3.3 & 6.3 & 5.6 & 0.04 \\
Cerebral vascular disease & 2.8 & 3.1 & 1.1 & 0.05 \\
Dementia & 0 & 0.2 & 9.3 & 0.02 \\
Chronic lung disease & 5.1 & 6.3 & 7.7 & 0.05 \\
Rheumatologic condition & 3.8 & 7.3 & 5.0 & 0.04 \\
Peptic ulcer disease & 1.8 & 4.0 & 31.9 & 0.04 \\
Diabetes & 24.1 & 39.2 & 3.7 & $<0.001$ \\
Diabetes with complications & 13.4 & 25.2 & 0.5 & 0.10 \\
Moderate/severe liver disease & 1.3 & 2.8 & 0.3 & 0.36 \\
Metastatic cancer & 1.3 & 0.6 & 1.3 & 0.45 \\
Leukemia & 0.5 & 0.2 & 17.2 & 0.06 \\
Lymphoma & 2.0 & 0.7 & 2.4 & 0.01 \\
Congestive heart failure & 10.0 & 14.9 & 2.7 & 0.94 \\
HIV & 2.8 & & & 0.3 \\
\hline
\end{tabular}

Percentages are presented in the table except for the CCI score.

HIV, human immunodeficiency virus; KT, kidney transplantation; CCI, Charlson comorbidity index; IQR, interquartile range.

$37.5 \%$ had a high comorbidity burden but were nonfrail and did not have ADL disability, while $41.8 \%$ had a low comorbidity burden, were nonfrail, and had no ADL disability. Only $1.2 \%$ had all 3 conditions present at time of KT evaluation.

Frail KT candidates had a greater burden of comorbidities (Table 2); the median CCI score was 2 (IQR 0-4) for those who were frail compared to 0 for those who were nonfrail (IQR $0-2 ; p<0.001$ ). Compared to nonfrail can- didates, frail and intermediate frail candidates had a higher prevalence of diabetes (frail: $51.9 \%$, intermediate frail: $39.2 \%$, and nonfrail: $24.1 \%, p<0.001$ ), diabetes with complications (frail: $37.3 \%$, intermediate frail: $25.2 \%$, and nonfrail: $13.4 \%, p<0.001$ ), congestive heart failure (frail: $17.2 \%$, intermediate frail: $14.9 \%$, and nonfrail: $10.0 \%, p=$ 0.01 ), and dementia (frail: $1.1 \%$, intermediate frail: $0.2 \%$, and nonfrail: $0 \%, p=0.02$ ). We also identified a higher prevalence of peripheral vascular disease, cerebral vascu- 
lar disease, chronic lung disease, rheumatologic disorder, and peptic ulcer in frail and intermediate frail groups compared to nonfrail patients.

\section{Comorbidity Burden and Risk of Waitlist Mortality}

KT candidates with a high comorbidity burden had a greater risk of waitlist mortality compared to those with a low comorbidity burden (log rank $p<0.001$; Fig. 2). Compared to KT candidates with a low comorbidity burden, those with a high comorbidity burden had 1.38 times the risk of waitlist mortality after adjusting for factors included in the registry-based model (95\% CI 1.01-1.89; Table 3). Similarly, each one-point increase in CCI score was associated with a 1.08 times increased risk of mortality after adjustment (95\% CI 1.03-1.13).

\section{Comorbidity Burden and Risk of Waitlist Mortality, by Frailty Status}

The risk of waitlist mortality associated with comorbidity burden differed by frailty status after adjusting for factors included in the registry-based model ( $p=0.01$; Table 3). Among nonfrail KT candidates, a high comorbidity burden was associated with a 1.66 times (95\% CI 1.172.35) greater risk of waitlist mortality. In contrast, among frail candidates, high comorbidity burden was not associated with waitlist mortality (HR 0.75, 95\% CI 0.44-1.29).

\section{Comorbidity Burden and the Risk of Waitlist \\ Mortality, by Candidate Age}

Furthermore, the risk of waitlist mortality associated with comorbidity burden differed by candidate age after adjusting for factors included in the registry-based model $(p=0.04)$. In younger KT candidates (age $<65$ years), a high comorbidity burden was associated with a 1.75 times $(95 \%$ CI 1.22-2.52) increased risk of waitlist mortality as compared to younger KT candidates without a high comorbidity burden. In contrast, among older KT candidates (age $\geq 65$ years), a high comorbidity burden was not associated with waitlist mortality (HR 0.97, 95\% CI 0.60-1.56).

\section{Improved Waitlist Mortality Risk Prediction}

The c-statistic for the registry-based model was 0.640. Adding the CCI score to the registry-based model increased the c-statistic to $0.657(p=0.02)$. Additionally, adding the synergistic effects of frailty and high comorbidity burden to the registry-based model for waitlist mortality, the c-statistic improved to $0.656(p<0.001)$; however, adding the synergistic effects of older age and high comorbidity burden did not improve prediction of waitlist mortality $(p=0.873)$.

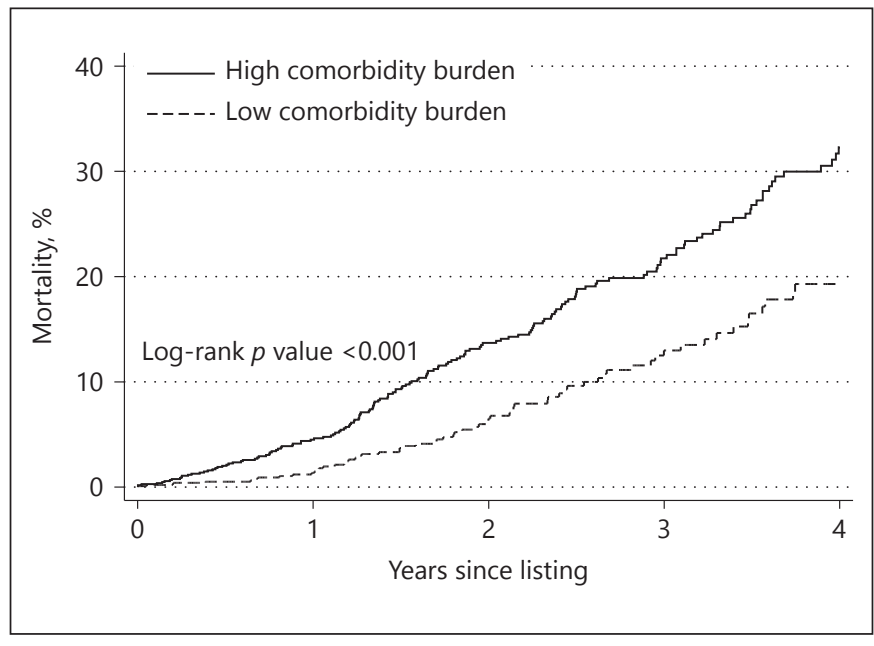

Fig. 2. Risk of waitlist mortality by CCI among KT candidates of all ages. We defined a high comorbidity burden as a CCI score of $\geq 2$ and low comorbidity burden as a CCI score of 0 or 1 .

Table 3. CCI and risk of mortality, by frailty, age, race and Gender among KT Candidates $(n=2,086)$. The CCI is modified for ESRD patients and we defined a high comorbidity burden as a CCI score of $\geq 2$ and low comorbidity burden as a CCI score of 0 or 1

\begin{tabular}{|c|c|c|}
\hline & \multicolumn{2}{|l|}{ Risk of mortality } \\
\hline & $\begin{array}{l}\text { 1-point increase } \\
\text { in CCI score, } \\
\text { HR }(95 \% \text { CI })\end{array}$ & $\begin{array}{l}\text { high } \\
\text { comorbidity } \\
\text { burden, } \\
\text { HR }(95 \% \mathrm{CI})\end{array}$ \\
\hline Overall risk & $1.08(1.03-1.13)$ & $1.38(1.01-1.89)$ \\
\hline \multicolumn{3}{|l|}{ Frailty } \\
\hline Nonfrail & $1.10(1.05-1.15)$ & $1.66(1.17-2.35)$ \\
\hline Frail & $1.02(0.92-1.12)$ & $0.75(0.44-1.29)$ \\
\hline$p$ for interaction & 0.16 & 0.01 \\
\hline \multicolumn{3}{|l|}{ Age } \\
\hline$<65$ & $1.10(1.05-1.15)$ & $1.75(1.22-2.52)$ \\
\hline$\geq 65$ & $1.02(0.92-1.13)$ & $0.97(0.60-1.56)$ \\
\hline$p$ for interaction & 0.16 & 0.04 \\
\hline \multicolumn{3}{|l|}{ Race } \\
\hline Non-African American & $1.13(1.06-1.21)$ & $1.72(1.16-2.55)$ \\
\hline African American & $1.04(0.97-1.11)$ & $1.03(0.67-1.59)$ \\
\hline$p$ for interaction & 0.31 & 0.06 \\
\hline \multicolumn{3}{|l|}{ Gender } \\
\hline Male & $1.10(1.05-1.15)$ & $1.53(1.05-2.24)$ \\
\hline Female & $1.02(0.93-1.13)$ & $1.17(0.74-1.85)$ \\
\hline$p$ for interaction & 0.17 & 0.33 \\
\hline
\end{tabular}

CCI, charlson comorbidity index; KT, kidney transplant; ESRD, end stage renal disease. 


\section{Discussion}

In this multicenter prospective, longitudinal study of 2,086 KT candidates, $51 \%$ of the cohort had a high burden of comorbidity, and it was more common among frail and intermediately frail KT candidates. The most common comorbidities were diabetes and congestive heart failure. There was a synergistic effect of frailty and a high comorbidity burden as well as older age and comorbidity on waitlist mortality such that a high comorbidity burden was only associated with waitlist mortality among candidates who were not frail and candidates who were younger. Furthermore, adding the synergistic effect of frailty and comorbidity, but not age and comorbidity, to the waitlist mortality model improved risk predication.

Our findings on the burden of comorbidity are consistent with previous studies of comorbidity in KT recipients in which $45 \%$ [8] to $47 \%$ [33] have a comorbidity, and we have extended these findings to a novel population of KT candidates. However, our results are higher than the previously reported $29 \%$ [5] found in one other study of KT candidates. These differences are expected as each study used different thresholds to define comorbidity and not all KT candidates go on to receive KT due to a high waitlist mortality or progressive disease. We extended the previous findings on an increased risk of the association between CCI score and mortality in patients undergoing dialysis $[34,35]$ and $\mathrm{KT}$ recipients $[5,8]$ to KT candidates. Only one study of a cohort of KT candidates from Andalusia, Spain (1984-2012) investigated the risk of waitlist mortality associated with CCI and found that a CCI of 3 or higher was associated with a 2.8fold increased risk of waitlist mortality [36]. Importantly, we have expanded this research to a more racially diverse population of KT recipients in the United States and further explored the association between comorbidity and frailty.

Furthermore, our findings extend the growing body of research on frailty among KT candidates and recipient to show that frailty is distinct from disability in this population [16-18, 21-28, 30, 31] by showing that among frail KT candidates, a high comorbidity burden is not associated with waitlist mortality. However, among nonfrail candidates, a high comorbidity burden is. These findings are consistent with the conceptual model for frailty in which this phenotype can result from comorbidity but also exist in the absence of comorbidity. A possible explanation is that, in frail waitlist patients, frailty itself is a greater risk factor for mortality than the presence of oth- er comorbid conditions, which may mean that frail patients may be further along in their disease progression and physiologic aging, as is suggested by studies of older adults with other comorbidities $[37,38]$. These results are supported by our finding and previously published results [6] suggesting that comorbidity was associated with waitlist mortality among younger, but not older, KT candidates. Finally, our findings suggest that measuring both frailty and comorbidity are important for improved risk prediction; we found that adding the synergistic effects of frailty and high comorbidity burden to the registry-based model for waitlist mortality, significantly improved the c-statistic from 0.640 to $0.656(p<0.001)$.

Strengths of this study included the comprehensive ascertainment of comorbidity and the prospective measurement of a validated, objective frailty instrument. Moreover, the study included 2,086 KT candidates of all ages, not just older adults and had long mean follow-up. Additionally, we were able to demonstrate statistically significant improvements in risk prediction by adding the synergistic effect of frailty and comorbidity to the registry model for waitlist mortality. The main limitation was that only 2 centers were included; however, the 2 centers are located in different regions and have different case mixes with respect to age and race.

In conclusion, in this prospective cohort study, KT candidates of all ages had a high comorbidity burden. Although comorbidity burden was associated with waitlist mortality, this risk was specific to nonfrail KT candidates. In fact, including both comorbidity and frailty in the national registry model significantly improved mortality risk prediction. While there was a synergistic effect of age and comorbidity on waitlist mortality, these factors did not improve mortality risk prediction. These results are important for clinicians to consider, as this study identifies vulnerable KT candidates who could benefit from targeted interventions such as prehabilitation to prevent waitlist mortality.

\section{Acknowledgments}

We would like to thank the participants of our study for participating in this important research study.

\section{Disclosure Statement}

The authors have no conflicts of interest to disclose. The results presented in this paper have not been published previously in whole or part, except in abstract format. 


\section{Author Contributions}

M.P.F., P.M.M., and C.E.H.: participated in research design, participated in the writing of the paper, and participated in the performance of the research. H.Y.: participated in research design, participated in the writing of the paper, and participated in data analysis. N.M.C., D.M.R.P., and L.R.-M.: participated in the writing of the paper. S.P.N., J.D.W., and D.L.S.: participated in the writing of the paper and participated in the performance of the research. M.A.M.-D.: participated in research design, participated in the writing of the paper, participated in the performance of the research, and participated in data analysis.

\section{Funding Source}

This study was supported by the National Institute on Aging grant numbers: F32AG053025 (PI: Haugen), R01AG042504(PI: Segev), K01AG043501 (PI: McAdams-DeMarco), R01AG055781 (PI: McAdams-DeMarco). This work was also supported by the National Institute of Diabetes and Digestive and Kidney Disease grant numbers: K24DK101828 (PI: Segev) and R01DK114074 (PI: McAdams-DeMarco). M.A.M.-D. was also supported by the Johns Hopkins University Claude D. Pepper Older Americans Independence Center (P30AG021334). The funders above did not have a role in study design; collection, analyses, and interpretation of data; writing the report; and decision to submit the report for publication.

\section{References}

1 Keane WF, Collins AJ: Influence of co-morbidity on mortality and morbidity in patients treated with hemodialysis. Am J Kidney Dis 1994;24:1010-1018.

2 Khan IH, Catto GR, Edward N, et al: Influence of coexisting disease on survival on renal-replacement therapy. Lancet 1993;341:415418.

3 Miskulin DC, Athienites NV, Yan G, et al: Comorbidity assessment using the Index of coexistent diseases in a multicenter clinical trial. Kidney Int 2001;60:1498-1510.

4 Hemmelgarn BR, Manns BJ, Quan H, et al: Adapting the Charlson comorbidity index for use in patients with ESRD. Am J Kidney Dis 2003;42:125-132.

5 Jassal SV, Schaubel DE, Fenton SS: Baseline comorbidity in kidney transplant recipients: a comparison of comorbidity indices. Am J Kidney Dis 2005;46:136-142.

6 Heldal K, Hartmann A, Leivestad T, et al: Clinical outcomes in elderly kidney transplant recipients are related to acute rejection episodes rather than pretransplant comorbidity. Transplantation 2009;87:10451051.

7 Levine MA, Schuler T, Gourishankar S: Complications in the 90 -day postoperative period following kidney transplant and the relationship of the Charlson comorbidity index. Can Urol Assoc J 2017;11:388-393.

$8 \mathrm{Wu}$ C, Evans I, Joseph R, et al: Comorbid conditions in kidney transplantation: association with graft and patient survival. J Am Soc Nephrol 2005;16:3437-3444.

9 Fried LP, Tangen CM, Walston J, et al: Frailty in older adults: evidence for a phenotype. J Gerontol A Biol Sci Med Sci 2001;56:M146M156.

10 Bandeen-Roche K, Xue QL, Ferrucci L, et al: Phenotype of frailty: characterization in the women's health and aging studies. J Gerontol A Biol Sci Med Sci 2006;61:262-266.

11 Bandeen-Roche K, Seplaki CL, Huang J, et al: Frailty in older adults: a nationally representative profile in the United States. J Gerontol A Biol Sci Med Sci 2015;70:1427-1434.
12 Boyd CM, Xue QL, Simpson CF, et al: Frailty, hospitalization, and progression of disability in a cohort of disabled older women. Am J Med 2005;118:1225-1231.

13 Boyle PA, Buchman AS, Wilson RS, et al: Physical frailty is associated with incident mild cognitive impairment in communitybased older persons. J Am Geriatr Soc 2010; 58:248-255.

14 Winograd CH, Gerety MB, Chung M, et al: Screening for frailty: criteria and predictors of outcomes. J Am Geriatr Soc 1991;39:778-784.

15 Fried LP, Ferrucci L, Darer J, et al: Untangling the concepts of disability, frailty, and comorbidity: implications for improved targeting and care. J Gerontol A Biol Sci Med Sci 2004; 59:255-263.

16 McAdams-DeMarco MA, Law A, King E, et al: Frailty and mortality in kidney transplant recipients. Am J Transplant 2015;15:149-154.

17 McAdams-DeMarco MA, Law A, Salter ML, et al: Frailty as a novel predictor of mortality and hospitalization in individuals of all ages undergoing hemodialysis. J Am Geriatr Soc 2013;61:896-901.

18 McAdams-DeMarco MA, Ying H, Olorundare I, et al: Individual frailty components and mortality in kidney transplant recipients. Transplantation 2017;101:2126-2132.

19 Fitzpatrick J, Sozio SM, Jaar BG, et al: Frailty, body composition and the risk of mortality in incident hemodialysis patients: the predictors of arrhythmic and cardiovascular risk in end stage renal disease study. Nephrol Dial Transplant 2018.

20 Konel JM, Warsame F, Ying H, et al: Depressive symptoms, frailty, and adverse Outcomes among kidney transplant recipients. Clin Transplant 2018:e13391.

21 McAdams DeMarco M, Olorundare I, Ying $\mathrm{H}$, et al: Frailty and post-kidney transplant health-related quality of life Transplantation 2017.

22 McAdams-DeMarco MA, Isaacs K, Darko L, et al: Changes in frailty after kidney transplantation. J Am Geriatr Soc 2015;63:21522157.
23 McAdams-DeMarco MA, King EA, Luo X, et al: Frailty, length of stay, and mortality in kidney transplant recipients: a national registry and prospective cohort study. Ann Surg 2016.

24 McAdams-DeMarco MA, Law A, Salter ML, et al: Frailty and early hospital readmission after kidney transplantation. Am J Transplant 2013;13:2091-2095.

25 McAdams-DeMarco MA, Law A, Tan J, et al: Frailty, mycophenolate reduction, and graft loss in kidney transplant recipients. Transplantation 2015;99:805-810.

26 McAdams-DeMarco MA, Olorundare IO, Ying $\mathrm{H}$, et al: Frailty and postkidney transplant health-related quality of life. Transplantation 2018;102:291-299.

27 McAdams-DeMarco MA, Suresh S, Law A, et al: Frailty and falls among adult patients undergoing chronic hemodialysis: a prospective cohort study. BMC Nephrol 2013;14:224.

28 McAdams-DeMarco MA, Tan J, Salter ML, et al: Frailty and cognitive function in incident hemodialysis patients. Clin J Am Soc Nephrol 2015;10:2181-2189.

29 Van Pilsum Rasmussen S, Konel J, Warsame $\mathrm{F}$, et al: Engaging clinicians and patients to assess and improve frailty measurement in adults with end stage renal disease. BMC Nephrol 2018;19:8.

30 Haugen CE, Mountford A, Warsame F, et al: Incidence, risk factors, and sequelae of postkidney transplant delirium. J Am Soc Nephrol 2018;29:1752-1759.

31 McAdams-DeMarco MA, Ying H, Thomas AG, et al: Frailty, inflammatory markers, and waitlist mortality among patients with endstage renal disease in a prospective cohort study. Transplantation 2018;102:1740-1746.

32 Harrell FE Jr, Lee KL, Mark DB: Multivariable prognostic models: issues in developing models, evaluating assumptions and adequacy, and measuring and reducing errors. Stat Med 1996;15:361-387.

33 Laging M, Kal-van Gestel JA, van de Wetering J, et al: A high comorbidity score should not be a contraindication for kidney transplantation. Transplantation 2016;100:400-406. 
34 Chae JW, Song CS, Kim H, et al: Prediction of mortality in patients undergoing maintenance hemodialysis by Charlson comorbidity index using ICD-10 database. Nephron Clin Pract 2011;117: c379-c384.

35 Fried L, Bernardini J, Piraino B: Charlson comorbidity index as a predictor of outcomes in incident peritoneal dialysis patients. Am J Kidney Dis 2001;37:337-342.

36 Hernandez D, de la Nuez PC, Muriel A, et al: Clinical assessment of mortality risk in renal transplant candidates in Spain. Transplantation 2014;98:653-659.

37 Castro-Rodriguez M, Carnicero JA, GarciaGarcia FJ, et al: Frailty as a major factor in the increased risk of death and disability in older people with diabetes. J Am Med Dir Assoc 2016;17:949-955.

38 Bonaga B, Sanchez-Jurado PM, MartinezReig M, et al: Frailty, Polypharmacy, and health outcomes in older adults: the frailty and dependence in Albacete study. J Am Med Dir Assoc 2018;19:46-52. 